\title{
Immune checkpoints inhibitors rechallenge in non-small-cell lung cancer: different scenarios with different solutions?
}

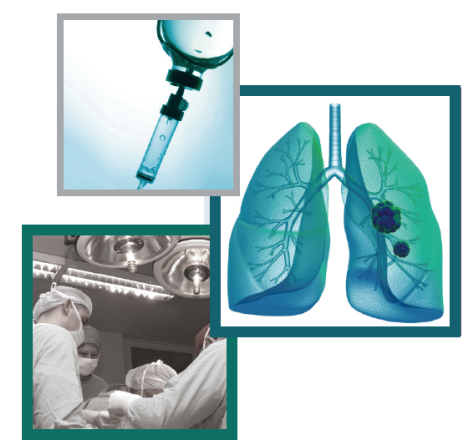

\author{
Giulio Metro*,1 (iD) \& Diego Signorelli² \\ ${ }^{1}$ Medical Oncology, Santa Maria della Misericordia Hospital, Azienda Ospedaliera di Perugia, Perugia, Italy \\ ${ }^{2}$ Medical Oncology Department, Fondazione IRCCS, Istituto Nazionale Tumori di Milano, Milano, Italy \\ *Author for correspondence: giulio.metro@yahoo.com
}

'6 it is unknown what should be the optimal duration of ICls treatment."

First draft submitted: 28 November 2019; Accepted for publication: 4 December 2019; Published online: 16 January 2020

Keywords: duration of treatment $\bullet$ immune checkpoint inhibitors $\bullet$ immunotherapy $\bullet$ non-small-cell lung cancer $\bullet$ rechallenge

In recent years, the oncologic community has witnessed a revolution in the treatment of patients with advanced non-small-cell lung cancer (NSCLC) and no actionable mutations [1]. For these patients, unleashing the immune response against cancer with the specific use of immune checkpoint inhibitors (ICIs) aimed at blocking the programmed cell death 1 (PD-1), programmed cell death ligand 1 (PD-L1) and anti-cytotoxic T lymphocyte antigen-4 (CTLA-4) has resulted into a dramatic improvement of overall prognosis. Recent data also suggest that, depending on the levels of PD-L1 expression on tumor cells, first-line therapy with an ICI, with or without cytotoxic chemotherapy, is associated with improved clinical outcome as compared with chemotherapy alone [2-4]. However, several questions still need to be addressed with regards to the optimal implementation of immunotherapy into routine clinical practice. First, it is still uncertain what is the value of adding cytotoxic chemotherapy to an ICI in patients with high PD-L1 expression $(\geq 50 \%)$ on tumor cells; second, there is an urgent need for biomarkers more reliable than PD-L1 expression for selection of patient candidates to immunotherapy; third, it is unknown what should be the optimal duration of ICIs treatment.

With regards to the latter point, it should be highlighted that a longer treatment duration is generally associated with a higher incidence of selected toxicities, namely immune-related adverse events (irAEs), which may negatively affect a patient's quality of life and even have life-threatening consequences. On the other hand, given the cost of immunotherapy, a shorter treatment duration may significantly decrease the burden of financial toxicity. Clinical trials have generally evaluated ICIs treatment for up to 2 years or more for responding patients. Importantly, few reasons suggest that benefits from immunotherapy may persist over time after stopping therapy, regardless of the reason for discontinuation. From a pharmacodynamic standpoint, it has been shown that receptor occupancy of PD-1 molecules on circulating $\mathrm{T}$ cells remains for almost 3 months after a single dose of nivolumab, despite a serum half-life of only 12-20 days [5]. Also, after three doses of nivolumab, occupancy remains at $40 \%$ for approximately 9 months since the last dose. Finally, response to immunotherapy has been largely attributed to the development of an adaptive immune response through the commitment of memory $\mathrm{T}$ cells within the tumor microenviroment [6]. Consistently, in one of the first trials that evaluated the anti-PD-1 agent pembrolizumab for patients with metastatic melanoma, an outstanding 2-year disease-free survival rate of $90 \%$ was reported for 67 out of the 105 complete responders who discontinued pembrolizumab and proceeded for observation without additional anticancer therapies [7]. Similarly, long-lasting responses that persist despite treatment discontinuation have been reported for NSCLC patients treated with an ICI [8]. In line with their persistent activity, delayed irAEs, meaning those manifesting $\geq 90$ days after discontinuation of immunotherapy, are being increasingly reported in the literature [9]. Certainly, this aspect also highlights the importance of prolonged vigilance for treatment-related adverse events in the post-ICI treatment setting. 
So far, the only trial that has prospectively addressed the question about the optimal duration of ICI treatment is CheckMate-153 [10]. In this study, patients with pretreated advanced NSCLC who were benefiting after 1 year of nivolumab in terms of either partial response or stable disease were randomized to continuous nivolumab versus observation followed by nivolumab retreatment at disease progression. The results clearly favored the continuous arm in terms of progression-free survival (PFS) from randomization (not reached vs 10.3 months, respectively, hazard ratio $=0.42[95 \%$ CI: $0.25-0.71]$ ), with a trend also toward improved overall survival (OS) (not reached vs 23.2 months, respectively, hazard ratio $=0.63$ [95\% CI: 0.33-1.20]). Even if PFS was only an exploratory end point (the primary end point was the incidence of high grade treatment-related adverse events), no central review of tumor assessment was performed and OS data are not mature yet, these results suggest that treatment duration in patients who are benefiting from an ICI should be certainly more than 1 year.

In clinical practice, immunotherapy rechallenge implies retreatment with agents aimed at blocking the anti-PD1 axis after a period of break. However, it is important to point out that patients who undergo immunotherapy rechallenge may represent a very heterogeneous population, especially in terms of the reason for prior ICI discontinuation. This issue should be taken into account when real-world data on patients subjected to immunotherapy rechallenge are critically revised. A large retrospective study on 1517 advanced NSCLCs found a remarkable median OS from the time of ICI rechallenge of 15.6 months [11]. However, this study did not report on the reason for prior immunotherapy discontinuation. On the other hand, another retrospective analysis by Gobbini et al. suggested that the efficacy of ICI rechallenge may greatly vary based on the cause of previous ICIs discontinuation. In fact, while these authors found a median OS from ICI rechallenge of 1.5 years, survival was higher in the subgroup of patients who discontinued the previous ICI treatment because of toxicity or clinical decision $(2.1$ and 1.5 years, respectively) and poor for those who had discontinued prior immunotherapy for disease progression (1 year) [12]. On this basis, we believe it is worthwhile that patients who undergo ICI rechallenge should be evaluated according to the three aforementioned clinical scenarios.

In one case, ICI rechallenge may refer to retreatment of a patient who previously discontinued immunotherapy because of an irAE. The decision of resuming immunotherapy in this situation clearly depends on the type and severity of the irAE that the patient had encountered. Recently, a retrospective study was conducted in order to address this relevant question [13]. The authors identified 68 out of 482 ICI-treated NSCLC patients who discontinued treatment because of an irAE, more commonly being pneumonitis, colitis, rash and liver enzymes abnormalities. A total of 38 patients were retreated with ICIs, of whom 18 patients (48\%) had no recurrence of irAE. On the other hand, ten patients (26\%) experienced recurrence of the same irAE, while another ten patients (26\%) developed a new irAE. Importantly, most of the recurrent/new irAEs were mild to moderate in intensity (12 out of 20,60\%), even though two treatment-related deaths were reported upon retreatment. On this basis, it seems that the decision on whether immunotherapy should be resumed in patients who discontinued it for toxicity must be carefully evaluated on a case by case basis, since as much as $50 \%$ of patients may experience a recurrent/new irAE. In this context, discontinuing ICI treatment for reasons other than disease progression might still be associated with long-term survival, as it has been recently shown in the publication of the long-term outcome results of advanced NSCLC patients treated with nivolumab within the Phase I CheckMate-003 study [14]. In this trial, of the 16 patients who survived $\geq 5$ years, only nine patients $(56.2 \%)$ had completed the planned 2-year course of nivolumab treatment, while the remainder seven patients $(43.8 \%)$ had terminated nivolumab earlier because of either disease progression $(n=3)$ or adverse events $(n=4)$. Against this background, it could be important to identify any risk factors that may guide the decision on whether ICI treatment should be resumed after discontinuation because of an irAE. A recent report conducted in advanced cancer patients who discontinued immunotherapy because of an immune-related colitis identified the following factors as those associated with a higher risk of recurrent colitis: need for immunosuppressive therapy with the first event, severity of colitis, longer duration of gastrointestinal symptoms and restart of anti-CTLA-4 therapy as opposed to anti-PD-(L)1 drugs [15].

In another case, immunotherapy rechallenge can be applied to patients who progress after terminating a prior course of ICIs treatment in the absence of disease progression. In this situation, ICIs rechallenge at the time of acquired resistance may reboost the expansion of memory $T$ cells against the tumor, which, in turn, could help to restore sensitivity to treatment. The benefits of ICIs rechallenge in this context have been retrospectively addressed in patients who were subjected to the anti-PD-L1 agent durvalumab within a Phase I/II study conducted across multiple tumor types [16]. Out of 1022 initial patients, 168 individuals completed the planned 1 year of treatment without disease progression, of whom 70 were retreated with durvalumab upon relapse. Overall, eight patients (11.4\%) responded to ICIs rechallenge, while 42 patients (60\%) experienced stable disease. Notably, out of the 
eight responding patients, five had responded to prior durvalumab, while on the total population of patients, the greatest benefit in terms of either partial response or stable disease was noted in individuals who had a treatmentfree interval since prior durvalumab $\geq 6$ months as compared with $<6$ months ( 87 vs $48 \%$ ). However, although this study has suggested a correlation between a long treatment-free interval and augmented sensitivity to ICIs rechallenge, it should be noted that the maximum duration of prior durvalumab was only 1 year, which should be considered suboptimal based on CheckMate-153 results [10]. Importantly, the specific outcome for the 21 NSCLC patients who were rechallenged with durvalumab within the same study was consistent with that of the overall population, with a PFS rate at 1 year of 31 versus $34.2 \%$, respectively. On the other hand, emerging data suggest that as much as two-thirds of NSCLC patients who have completed 2 years of pembrolizumab without disease progression, may experience clinical benefit upon retreatment with the same ICI at disease relapse [17,18]. In fact, retreatment with pembrolizumab in the KEYNOTE-010 study of platinum-pretreated patients with PD-L1 $\geq 1 \%$ and in the KEYNOTE-024 trial of chemonaive patients with PD-L1, $\geq 50 \%$ led to a partial response or stable disease in 11 out of 14 patients (78.5\%) and seven out of ten patients (70\%), respectively. At the present time, a parallel-group Phase II clinical trial is being conducted in order to test rechallenge with pembrolizumab in patients with acquired resistance after stopping treatment (NCT03526887).

Finally, in another situation, ICIs rechallenge can be applied to patients who progressed during treatment or within 12 weeks of termination of immunotherapy. In this clinical scenario, ICIs rechallenge should be pursued only within clinical trials and several studies are ongoing in order to address different strategies of rechallenge such as: retreatment after intervening chemotherapy (NCT03526887), rechallenge with the addition of an antiCTLA-4 agent to an anti-PD-1 drug (NCT03262779), retreatment with continuation of anti-PD-1 agent beyond progression with the concomitant administration of a cytotoxic agent not previously administered (NCT03041181) and rechallenge with the addition of a targeted therapy to an ICI (NCT03334617, NCT03829332). Importantly, most of the aforementioned clinical trials include a mandatory rebiopsy at baseline in order to select the most appropriate treatment and/or understand the molecular mechanisms underlying resistance to ICI treatment.

To conclude, we believe that the exact duration of ICIs treatment and the benefit of ICIs rechallenge for advanced NSCLC are still poorly defined. While a duration of at least 2 years for ICIs treatment has been suggested in a clinical trial, the evidence on the benefit of ICIs rechallenge has been mainly obtained from retrospective studies. However, we suggest that in case of discontinuation because of an irAE, immunotherapy rechallenge can be considered at resolution of toxicity only in selected cases based on the type, severity and duration of the irAE. On the other hand, in case of progression after terminating a prior course of ICIs treatment, rechallenge can be considered, especially after a treatment-free interval $\geq 6$ months based on whether there is a lack of valid therapeutic alternatives. Finally, ICIs rechallenge after an intervening treatment in patients who progressed during immunotherapy should be regarded as investigational and enrollment in clinical trials is highly encouraged.

Notwithstanding, regardless of whether progression occurred during or after termination of ICIs treatment, only the understanding of the molecular mechanisms behind ICIs resistance would help to find novel targets, either immune or biological ones, which eventually may facilitate the development of therapeutic strategies that could extend the proportion of patients benefiting from immunotherapy rechallenge through the use of combinatorial approaches.

Financial \& competing interests disclosure

G Metro discloses consultancies from Boehringer Ingelheim and Bristol-Myers Squibb and travel grants from AstraZeneca. D Signorelli discloses a consultancy from AstraZeneca and travel grants from Bristol-Myers Squibb, Merck, Pfizer and Roche. The authors have no other relevant affiliations or financial involvement with any organization or entity with a financial interest in or financial conflict with the subject matter or materials discussed in the manuscript apart from those disclosed.

No writing assistance was utilized in the production of this manuscript.

Open access

This work is licensed under the Attribution-NonCommercial-NoDerivatives 4.0 Unported License. To view a copy of this license, visit http://creativecommons.org/licenses/by-nc-nd/4.0/ 


\section{References}

1. Toschi L, Rossi S, Finocchiaro G, Santoro A. Non-small-cell lung cancer treatment (r)evolution: ten years of advances and more to come. Ecancermedicalscience 11, 787 (2017).

2. Addeo A, Banna GL, Metro G, Di Maio M. Chemotherapy in combination with immune checkpoint inhibitors for the first-line treatment of patients with advanced non-small-cell lung cancer: a systematic review and literature-based meta-analysis. Front. Oncol. 9 , 264 (2019).

3. Reck M, Rodríguez-Abreu D, Robinson AG et al. Pembrolizumab versus chemotherapy for PD-L1-positive non-small-cell lung cancer. N. Engl. J. Med. 375(19), 1823-1833 (2016).

4. Spigel D, de Marinis F, Giaccone G et al. Impower110: interim overall survival (OS) analysis of a phase III study of atezolizumab (atezo) vs platinum-based chemotherapy (chemo) as first-line (1L) treatment (tx) in PD-L1-selected NSCLC. Ann. Oncol. 30(Suppl. 5), v851-v934 (2019).

5. Brahmer JR, Drake CG, Wollner I et al. Phase I study of single-agent anti-programmed death-1 (MDX-1106) in refractory solid tumors: safety, clinical activity, pharmacodynamics, and immunologic correlates. J. Clin. Oncol. 28(19), 3167-3175 (2010).

6. Edwards J, Wilmott JS, Madore J et al. CD103 ${ }^{+}$tumor-resident $\mathrm{CD} 8^{+} \mathrm{T}$ cells are associated with improved survival in immunotherapy-naïve melanoma patients and expand significantly during anti-PD-1 treatment. Clin. Cancer Res. 24(13), 3036-3045 (2018).

7. Robert C, Ribas A, Hamid O et al. Durable complete response after discontinuation of pembrolizumab in patients with metastatic melanoma. J. Clin. Oncol. 36(17), 1668-1674 (2018).

8. Gettinger SN, Horn L, Gandhi L et al. Overall survival and long-term safety of Nivolumab (anti-programmed death 1 antibody, BMS-936558, ONO-4538) in patients with previously treated advanced non-small-cell lung cancer. J. Clin. Oncol. 33(18), 2004-2012 (2015).

9. Couey MA, Bell RB, Patel AA et al. Delayed immune-related events (DIRE) after discontinuation of immunotherapy: diagnostic hazard of autoimmunity at a distance. J. Immunother. Cancer 7(1), 165 (2019).

10. Spigel DR, McCleod M, Hussein MA et al. CheckMate 153: randomized results of continuous vs 1-year fixed-duration nivolumab in patients with advanced non-small-cell lung cancer. Ann. Oncol. 28(Suppl. 5), v460-v496 (2017).

11. Giaj-Levra M, Cottè FE, Corre R et al. Immunotherapy rechallenge after nivolumab treatment in advanced non-small-cell lung cancer in the French real-world setting. J. Thorac. Oncol. 14(Suppl.10), S274-275 (2019).

12. Gobbini E, Toffart A, Perol M et al. Immune checkpoint inhibitors re-challenge: outcomes analysis in a French national cohort of non-small-cell lung cancer patients. J. Thorac. Oncol. 14(Suppl. 10), S274 (2019).

13. Santini FC, Rizvi H, Plodkowski AJ et al. Safety and efficacy of re-treating with immunotherapy after immune-related adverse events in patients with NSCLC. Cancer Immunol. Res. 6(9), 1093-1099 (2018).

14. Gettinger S, Horn L, Jackman D et al. Five-year follow-up of nivolumab in previously treated advanced non-small-cell lung cancer: results from the CA209-003 study. J. Clin. Oncol. 36(17), 1675-1684 (2018).

15. Abu-Sbeih H, Ali FS, Naqash AR et al. Resumption of immune checkpoint inhibitor therapy after immune-mediated colitis. J. Clin. Oncol. 37(30), 2738-2745 (2019).

16. Sheth S, Gao C, Angra N, Mueller N, Martinez P, Soria JC. Durvalumab activity in previously treated patients who stopped durvalumab without disease progression. Ann. Oncol. 30(Suppl. 5), v475-v532 (2019).

17. Herbst RS, Garon EB, Kim DW et al. Long-term survival in patients (pts) with advanced NSCLC in the KEYNOTE-010 study overall and in pts who completed 2 years of pembrolizumab (pembro). Ann. Oncol. 29(Suppl. 8), mdy 424.075 (2018).

18. Reck M, Rodriguez-Abreu D, Robinson AG et al. KEYNOTE-024 3-year survival update: pembrolizumab versus platinum-based chemotherapy for advanced NSCLC. 2019 World Conference on Lung Cancer. Barcelona, Spain, 7-10 October, (2019). 